\title{
Transplanting heterotaxy...out with the vague, and in the with new
}

\author{
Aaron Eckhauser, MD, MS
}

\author{
From the Division of Cardiothoracic Surgery, Department of Surgery, University of Utah, Salt Lake City, Utah. \\ Disclosures: Author has nothing to disclose with regard to commercial support. \\ Received for publication Nov 22, 2018; revisions received Nov 22, 2018; accepted for publication Nov 26, 2018. \\ Address for reprints: Aaron Eckhauser, MD, MS, 100 N. Mario Capecchi Dr, Suite 2200, Salt Lake City, UT \\ 84113 (E-mail: Aaron.eckhauser@hsc.utah.edu). \\ J Thorac Cardiovasc Surg 2019;157:741-2 \\ $0022-5223 / \$ 36.00$ \\ Copyright (c) 2018 by The American Association for Thoracic Surgery \\ https://doi.org/10.1016/j.jtcvs.2018.11.075
}

Studying the clinical implications of heterotaxy syndrome in patients with congenital heart disease has been challenging and often relegated to the realm of case reports and single-center experiences. In this issue of the Journal, Duong and colleagues ${ }^{1}$ present the largest analysis to date studying the attendant risks and costs associated with children with heterotaxy syndrome who undergo transplantation. The power of this study lies in the unique linkage of the Scientific Registry of Transplant Recipients and the Pediatric Health Information System databases to analyze more than 180 patients with heterotaxy syndrome or situs anomalies undergoing heart transplant. Interestingly, they found that patients with heterotaxy syndrome or other situs anomalies have decreased survival after heart transplant (short and long term) and require greater amounts of resource use. In addition, these patients also tend to have longer ischemic times despite similar or shorter travel times, more cardiac reoperations, catheterizations with peripheral stenting, and renal failure compared with patients without heterotaxy undergoing transplantation.

This study deserves attention because it represents the first incidence of harnessing the power of big data using previously established linkage methodologies to study heterotaxy in pediatric transplantation recipients. ${ }^{2,3}$ Importantly, this study also highlights some of the limitations of using big data; namely, how cases are identified in differing types of databases, whether both inpatient and outpatient encounters are recorded, and if/how patients are tracked across hospitals.

The authors note that they were limited in their cohort identification by International Classification of Diseases codes, which can be ambiguous and nondiscriminatory, resulting in a heterogenous and not perfectly delineated cohort. Significant effort has been made to create a globally accepted nomenclature system for congenital heart disease (International Paediatric and Congenital Cardiac Code), which is used in the both the Society of Thoracic Surgeons

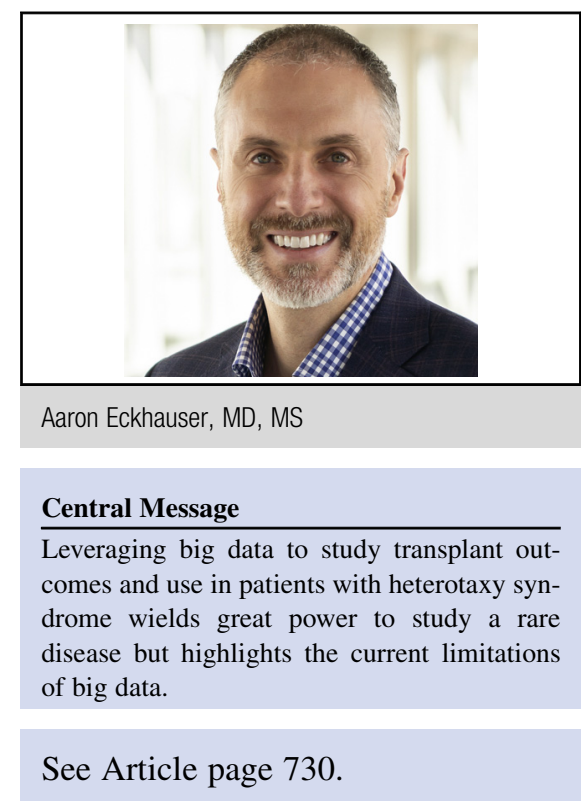

and European Association for Cardio-Thoracic Surgery congenital surgical databases. ${ }^{4}$ Unfortunately, administrative databases typically rely on the International Classification of Diseases diagnostic codes, which are not as accurate and discriminatory as the International Paediatric and Congenital Cardiac Code. In addition, we often rely on these linkages to "fill in" the gaps left by a lack of communication between electronic medical records, inpatient and outpatient visits, and inter- and intrastate admissions across facilities.

Although we are on the cusp of being able to really leverage the power of these large databases, we still have hurdles to overcome. In his 2016 response to the Health Care Payment-Learning Action Networks Data Sharing whitepaper, former Society of Thoracic Surgeons president Joe Bavaria outlined the obstacles to effective data sharing among qualified clinical data registries; (1) lack of access to federal and private payer claims data; (2) lack of access to death information, namely the Social Security Death Master File; (3) incomplete or inaccurate electronic health records data; and (4) inability to use unique device identifiers to map encounters across different systems.

Despite the inherent limitations of large databases, this excellent study shows how we can leverage this information to answer difficult questions in small populations of patients with congenital heart disease. Whether prognostically or 
programmatically, the question looms, how will these data inform our practice moving forward?

\section{References}

1. Duong SQ, Godown J, Soslow JH, Thurm C, Hall M, Sainathan S, et al. Increased mortality, morbidities, and costs after heart transplantation in heterotaxy syndrome and other complex situs arrangements. J Thorac Cardiovasc Surg. 2019;157: $730-40$.
2. Pasquali SK, Jacobs JP, Shook GJ, O’Brien SM, Hall M, Jacobs ML, et al. Linking clinical registry data with administrative data using indirect identifiers: implementation and validation in the congenital heart surgery population. Am Heart J. 2010; 160:1099-104.

3. Godown J, Thurm C, Dodd DA, Soslow JH, Feingold B, Smith AH, et al. A unique linkage of administrative and clinical registry databases to expand analytic possibilities in pediatric heart transplantation research. Am Heart J. 2017;194:9-15.

4. Jacobs JP, Mavroudis C, Jacobs ML, Maruszewski B, Tchervenkov CI, LacourGayet FG, et al. Nomenclature and databases - the past, the present, and the future: a primer for the congenital heart surgeon. Pediatr Cardiol. 2007;28:105-15. 